Check for updates

Cite this: J. Mater. Chem. B, 2020, 8, 8207

Received 25th June 2020

Accepted 7th August 2020

DOI: 10.1039/d0tb01576h

rsc.li/materials-b

\title{
Advancements in the development on new liquid embolic agents for use in therapeutic embolisation
}

\author{
Jasmine Lord, ${ }^{a}$ Hugh Britton, ${ }^{b}$ Sebastian G. Spain (D)*a and Andrew L. Lewis ${ }^{b}$
}

\begin{abstract}
Liquid formulations have a well-established role in therapeutic embolisation of blood vessels with the widespread use of cyanoacrylate glues, precipitating polymer suspensions, sclerosing agents and viscous emulsions of oil and chemotherapeutic agents. There is currently an emerging market for next generation liquid embolics which aim to address some of the short-comings of the currently used products. These next generation systems use varying chemistries in their approach to formulate new systems including polymerising, precipitating and phase-transitioning mechanisms to form solidified masses in situ within the vasculature. Some of these emerging technologies have been developed to possess improved imaging properties such as inherent radiopacity, rather than relying on having to mixing with radiopaque materials such as tantalum powder and reduction of X-ray imaging artefacts (streaking). Others offer solvent-free formulations which gel on contact with blood thereby allowing precise control over gel formation during the embolisation process without the use of potentially toxic solvents. In this review, we discuss the role of liquid agents in therapeutic embolisation and the potential of emerging technologies under development for use in the next generation of embolics.
\end{abstract}

\section{Introduction}

Therapeutic embolisation is a well-established treatment with a wide variety of embolic devices available on the market including coils, balloons and particles. The range of commercially available embolic agents represents the breadth of embolisation treatments that are performed and highlights the need to tailor each procedure on a case-by-case basis, dependent upon the size and extent of vasculature to be blocked and the need to block more proximal or distal to the position of the catheter through which they are delivered. There may, however, be complications with these techniques, some dependent upon the skill and experience of the physician and others that are influenced by the properties of the embolic agents. For example, microcatheter blocking $^{1}$ and off-target embolisations ${ }^{2}$ are the primary issues faced and can be a result of both of human error and poor handling characteristics of the embolic agent. Liquid embolic materials are no different in this respect but the ability to solidify in situ offers alternative characteristics to the more commonly used particulate systems that, although delivered in a liquid carrier, are preformed particles that are not colloidally stable,

\footnotetext{
${ }^{a}$ Department of Chemistry, University of Sheffield, Dainton Building, Brook Hill, Sheffield, S3 7HF, UK. E-mail: s.g.spain@sheffield.ac.uk

${ }^{b}$ Biocompatibles UK Ltd (a BTG International group company), Lakeview, Riverside Way, Watchmoor Park, Camberley, GU15 3YL, UK
}

and provides additional tools and capabilities to the treating physician for therapeutic embolisation applications.

Those liquid embolic agents that transition in phase function by solidification in situ when injected into the vasculature, changing from liquid to solid form by processes such as precipitation or polymerisation. The in situ formation of a gel enables the liquid formulation to be easily delivered using a microcatheter provided that the liquid materials are of an appropriate viscosity when in their solution state and circumvents the problem of microcatheter blockage due to aggregation of particles or microspheres.

Once the liquid undergoes a sol-gel transition, it provides an added advantage over preformed materials, such as coils, in that the resulting gelling mass can mould itself to fill the geometry of the vessel into which it is injected along with any irregularities. Hence, the final gel form is unique and conforms ideally to the contours of the vessel being treated. It acts to fully occlude the vessel and does not rely on thrombus formation to complete the occlusion. Therefore, liquid embolic materials are advantageous in patients with coagulopathies or patients receiving anti-coagulation therapy in which their ability to form blood clots is impaired. ${ }^{3,4}$ Furthermore, the formation of a single solid mass of embolic material at the treatment site also provides the advantage of a reduced risk of fragment migration once implanted. This is often a concern with embolic materials, such as particulates, which pose the risk of dislodging once implanted leading to off-target embolisation. 


\section{Established liquid embolics}

Excluding sclerosants that chemically damage the vessel wall in order to induce its collapse, there are currently three main liquid agents used for embolisation procedures in the clinic; cyanoacrylate glues (such as Trufill ${ }^{\circledR}$, Codman), ethylene-vinyl alcohol (EVA) copolymer solution (such as Onyx ${ }^{\circledR}$, Medtronic) and iodinated poppyseed oil (Lipiodol ${ }^{\circledR}$, Guerbet). Lipiodol ${ }^{\circledR}$ has been used for many years as an imaging agent and is used off-label in the treatment of heptatocellular carcinoma (HCC), where is it mixed with aqueous chemotherapy solutions to create a viscous emulsion that aids in temporarily reducing blood flow. ${ }^{5}$ It does not however, cause a permanent stasis of flow in the vessel. The cyanoacrylate glues and EVA solutions are used in a much wider range of vascular conditions but again, often in an off-label setting, including the treatment of arteriovenous malformations (AVMs), ${ }^{6}$ gastrointestinal bleeding $^{7}$ and aneurysms. ${ }^{8}$ These liquid embolics are primarily delivered using narrow lumen microcatheters with hydrophilic coatings that provide lubricity and reduce interactions with the inner lumen to aid the delivery of the liquids as they pass through the microcatheter during injection. In the case of EVA solutions that are formulated in the organic solvent dimethyl sulfoxide (DMSO), the microcatheter must be made of chemically inert materials to avoid being softened or dissolved by this powerful solvent. Balloon microcatheters are frequently utilised in the delivery of liquid embolics due to their ability to reduce blood flow during injection of the solidifying liquid thereby providing more control over the delivery process. The balloon is inflated behind the distal tip of the microcatheter in order to prevent blood flow past the tip, allowing the precipitation process to occur at the tip of the catheter as the solution mixes with the blood, reducing the risk of material fragmentation and stringing.

\subsection{Cyanoacrylate glues}

There are a number of glues commercially available including TruFill $^{\mathbb{R}}$ (Codman), Histoacryl ${ }^{\mathbb{R}}$ (B. Braun) and Glubran ${ }^{\circledR} 2$ (GEM) amongst many others. A recent entry to the market has been Sapheon VenaSeal ${ }^{\mathrm{TM}}$ (Medtronic Inc.) specifically indicated for the closure of the Greater Saphenous Vein (GSV) to treat varicose veins. The glue formulations are predominantly based on the monomer $N$-butyl-2-cyanoacrylate ( $n \mathrm{BCA})$, a derivative of Superglue $^{\mathrm{TM}}$. Other cyanoacrylate derivatives have been developed and tested in which the aliphatic side chain has been varied which affects rate of polymerisation and physical properties of the resulting polymer mass. There were some reports that the $N$-butyl version caused sarcomas and hence the isobutyl derivative has become favoured and most commonly used. ${ }^{9,10}$ On entering blood vessels, the $n$ BCA monomer rapidly polymerises under the ionic conditions found in blood to form an embolus within the vasculature (Fig. 1). Care must be taken during the preparation of the glue to prevent premature polymerisation of the monomer on contact with ionic solutions. Prior to the injection of glue, the microcatheter must be flushed with a non-ionic 5\% dextrose solution in order to prevent polymerisation within the microcatheter which could result in microcatheter blockage. Delivery of glue through pre-flushed microcatheters ensures that the glue rapidly polymerises on contact with blood upon exiting the tip of the microcatheter at the delivery site. The fast rate of polymerisation is particularly

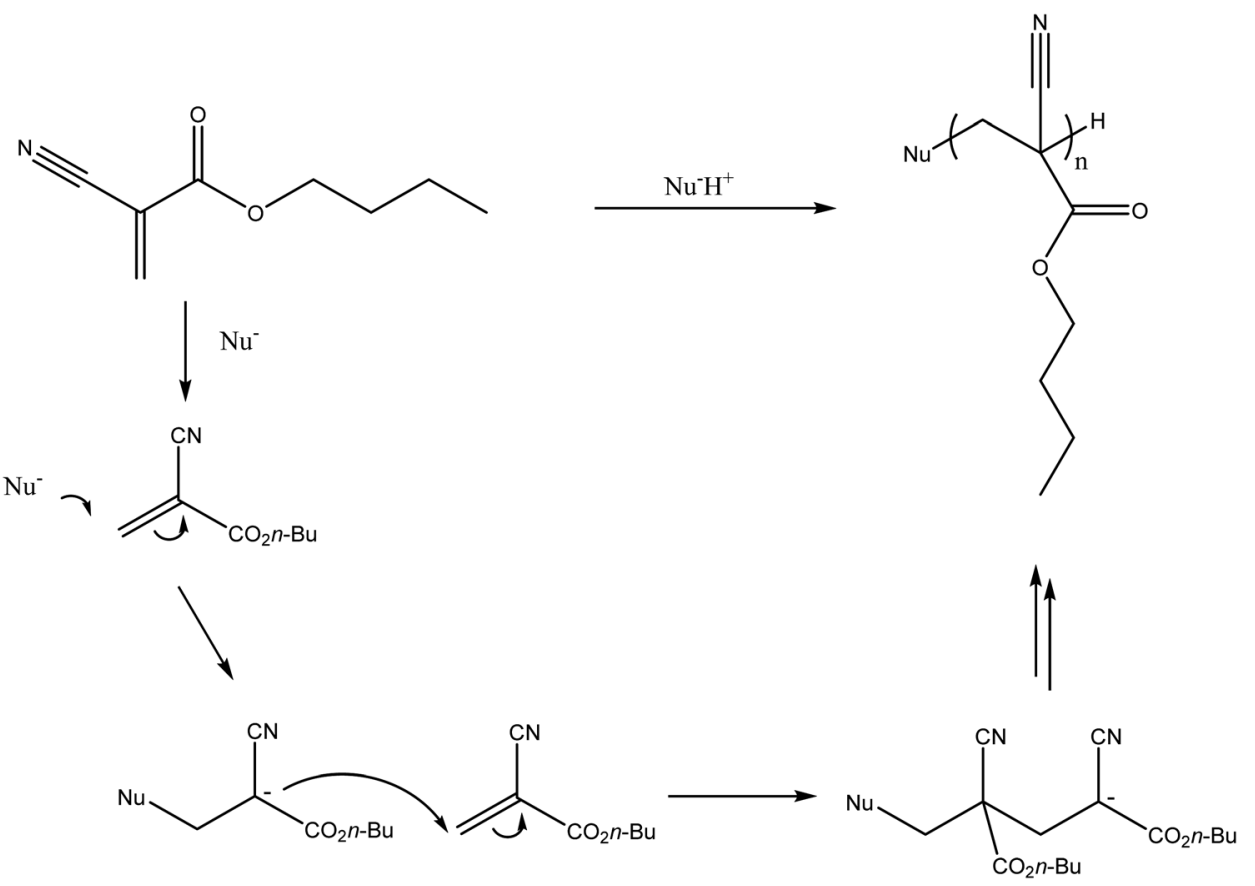

Fig. 1 In situ polymerisation of $\mathrm{N}$-butyl-2-cyanoacrylate ( $n \mathrm{BCA})$. ( $\mathrm{Nu}^{-}$refers to a nucleophilic species, which would most commonly be water in the case of delivery into the blood stream). 
beneficial in cases where rapid embolisation is required such as trauma or gastrointestinal bleeding.

The low viscosity nature of the $n$ BCA glue results in the formation of a solid cast within the blood vessels with occlusion focused distally. ${ }^{11}$ Embolisation with this system provides permanent occlusion of the targeted vessels due to the initiation of an acute inflammatory response in the blood vessel walls and surrounding tissue. This is thought to be a consequence of the polymerising species reacting with proteins and cellular structures in the vessel wall, the local generation of heat and potential to release toxic by-products such as formaldehyde during the polymerisation..$^{10,12,13}$ This leads to fibrosis of the surrounding area resulting in permanent occlusion of the treated blood vessels. ${ }^{14}$

The rate of polymerisation can be predictably controlled by the addition of glacial acetic acid and Lipiodol ${ }^{\circledR}$ (Guerbet Llc.) which act as polymerisation retardants. ${ }^{15}$ The addition of these polymerisation retardants to create dilutions in the range $25-67 \%$ is recommended as a method to slow the rate of polymerisation due to the reduced ionic contact of the glue with blood; $67 \% n \mathrm{BCA}$ will polymerised in around a second whereas $25 \% n$ BCA takes around 6 seconds. ${ }^{11}$ This provides greater flexibility in the working times of the glue. ${ }^{16}$ The use of Lipiodol ${ }^{\mathrm{R}}$ in combination with glue increases the overall viscosity of the liquid to improve control of the injection and reduce distal embolisation. Additionally, the Lipiodol ${ }^{\circledR}$ contrast agent has the advantage of imparting radiopacity to the embolic solution enabling the injection to be monitored by real-time fluoroscopy, although Trufill ${ }^{\mathbb{R}}$ glue kits contain both ethiodised oil and tantalum powder if additional radiopacity is desired.

Following the delivery of glue, it is necessary to immediately withdraw the microcatheter. This is to prevent the entrapment of the microcatheter at the site of administration due to the highly adhesive nature of the material. ${ }^{17}$ Gluing of the microcatheter in place may also occur due to excessive degrees of reflux of the material which is dependent on the volume injected or early polymerisation. Hence, careful attention must be made to the preparation of the liquid embolic prior to procedure, the delivery technique and the volume of liquid used. Intermittent injection of the polymerising liquid embolic can result in microcatheter blockage which requires the removal and placement of a new microcatheter when this occurs. ${ }^{18}$ The occurrence of microcatheter entrapment requires the microcatheter to be broken to leave the distal end in place within the vasculature. ${ }^{19}$ It is generally attempted to keep the remaining microcatheter length as short as possible to avoid the proximal end from blocking or rupturing nearby blood vessels. However, if this cannot be achieved then the microcatheter is cut at the point where it enters the artery and is then secured at the site of entry located at either the groin if femoral access was used or the wrist if radial access was used. ${ }^{20}$

Microcatheters with a hydrophilic coating have been shown to demonstrate reduced adhesion towards cyanoacrylate glue. ${ }^{21}$ Additionally, microcatheters are available with detachable tips, such as Apollo ${ }^{\mathrm{TM}}$ marketed by Medtronic Inc., which allow a short section of the microcatheter tip to be safely detached and left in place if adhesion to the embolic material were to occur. $^{22,23}$ However, the use of these microcatheters in the clinic must be carefully considered due to the resulting higher cost. The risk of microcatheter entrapment is largely dependent on the operator's proficiency and embolic material formulation hence specially designed microcatheters offer limited benefits. Despite the risks of microcatheter blocking and entrapment, $n$ BCA glue is still in widespread clinical use, particularly due to its low cost, with interventional radiologists that are experienced and proficient in its delivery.

\subsection{Ethylene vinyl alcohol copolymer, Onyx ${ }^{\circledR}$}

Onyx ${ }^{\circledR}$ (Medtronic Inc.) is a liquid embolic system based on a poly(ethylene-co-vinyl alcohol) (EVA) copolymer (Fig. 2) dissolved in DMSO with micronised tantalum powder added in suspension. Prior to delivery, Onyx ${ }^{\circledR}$ must be agitated for a minimum of 20 minutes to achieve a uniform suspension of the DMSO polymer solution and tantalum radiopacifying agent as the metal particles sediment quickly over time due to their density. $^{24}$ On delivery into the vasculature, the polymer precipitates out of solution as it is diluted by the aqueous conditions of blood which act as a non-solvent for the polymer. The precipitation of the polymer results in the entrapment of the suspended tantalum powder and the gradient diffusion of DMSO from the precipitated plug. Once precipitated, Onyx ${ }^{\circledR}$ forms a gel with a sponge-like consistency making it ideal for use in the treatment of AVMs as the occluded vessels are soft and easily removed by surgical methods. ${ }^{25,26}$ The tantalum contained within the precipitated gel imparts long-term radiopacity to the material meaning the embolised vessels can be easily located by X-ray imaging. The suspended tantalum imparts a black colour to the suspension hence care must be taken when treating sites near to the skin surface as this can show through the skin layers and cause a tattoo-like staining which can also be observed internally by endoscopy (Fig. 3 and 4). In addition, it is important to be cognisant of the location of a previously treated site when considering follow-on therapies, as application of treatments such as radiofrequency ablation in the vicinity of the occluded mass can induce sparking between the metal particles.

Onyx ${ }^{\circledR}$ is marketed with a range of different viscosities including Onyx ${ }^{\circledR} 18$ which is $18 \mathrm{mPa} \mathrm{s}$, Onyx ${ }^{\circledR} 34$ which is $34 \mathrm{mPa} \mathrm{s}$ and Onyx ${ }^{\circledR} 500$ which is $500 \mathrm{mPa} \mathrm{s}$. The different Onyx ${ }^{\circledR}$ formulations available allow the selection of appropriate viscosities depending on the size and blood flow rate in the vasculature targeted. For example, low viscosity Onyx ${ }^{\circledR} 18$ is suited for treatments requiring high degrees of distal penetration, such as AVMs, as the low viscosity solution can penetrate deep into the nidus of the growth. ${ }^{29}$ Whereas, high viscosity Onyx ${ }^{\circledR} 500$ is better suited for embolisation of aneurysms,

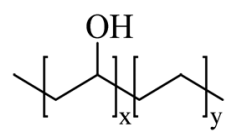

Fig. 2 Chemical structure of poly(ethylene-co-vinyl alcohol). 

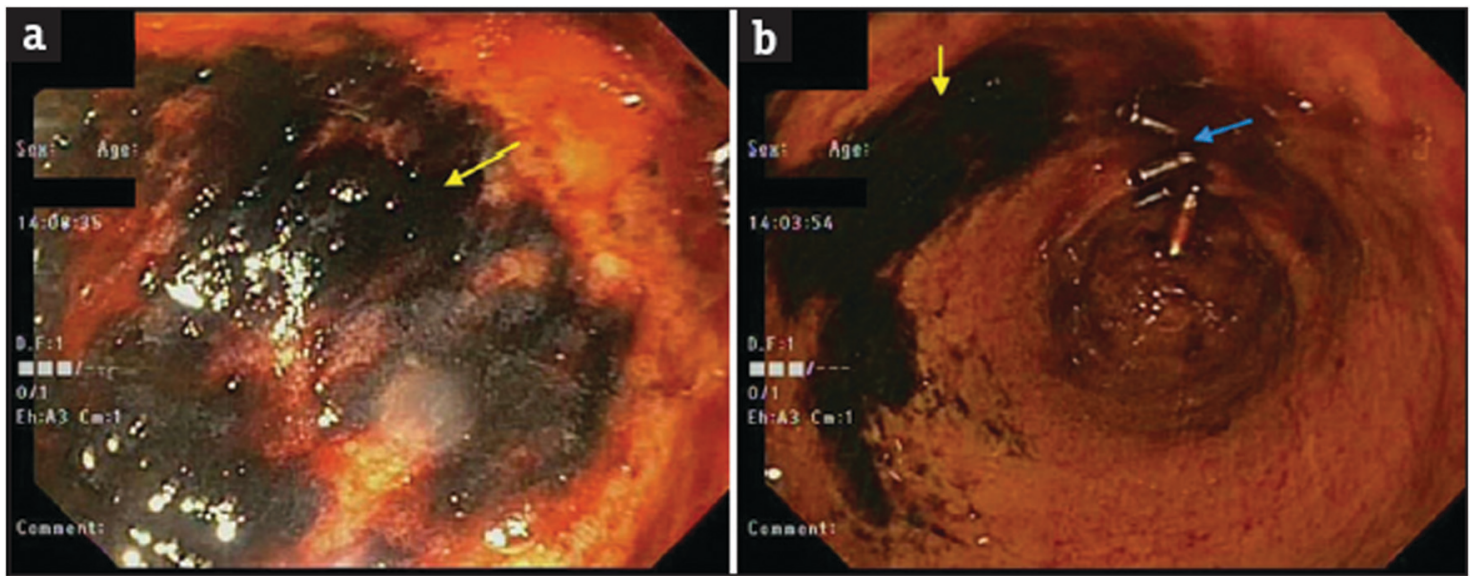

Fig. 3 Endoscopy after embolisation of the right gastric artery branches with Onyx ${ }^{\mathbb{R}}$ demonstrating black staining/discoloration of the mucosa in the prepyloric region with relatively normal surrounding gastric mucosa (yellow) next to hemoclips (blue arrows). Reproduced with permission from J. Clin. Imag. Sci. 2018, 8, 46. ${ }^{27}$

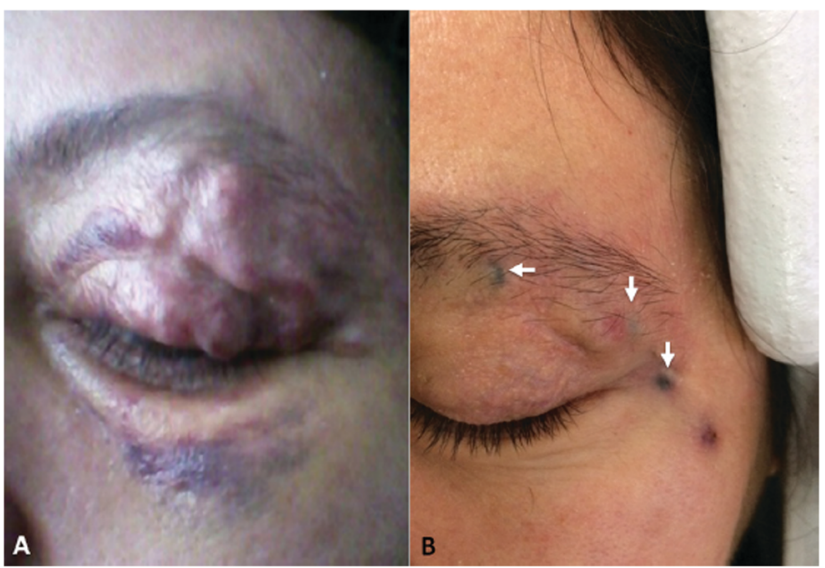

Fig. 4 Showing the tattooing of effect Onyx ${ }^{\circledR} 18$ on an upper eyelid pulsating mass pre-(A) and post-(B) treatment. Reproduced from J. Neurolnt. Surg. $2018,10,240-244 .{ }^{28}$

particularly of wide necks which are not suitable for surgical clipping, as the high viscosity can resist potential wash-out of the material due to the flow of blood past the aneurysm neck. ${ }^{30}$

In contrast to $n \mathrm{BCA}$ glue, Onyx ${ }^{\mathbb{R}}$ does not initiate any significant inflammatory response. However, injection of the organic solvent DMSO necessitates further patient sedation to reduce discomfort. The solvent also penetrates through the skin and results in an unpleasant garlic-like odour. ${ }^{31}$ Injection of DMSO can also result in the occurrence of vasospasm, a phenomenon in which the blood vessels suddenly constrict causing reduced diameters and blood flow, due to the cytotoxicity of the solvent. ${ }^{32}$ If this occurs then the injection must be halted until the vasospasm stops which increases the procedural time and patient risks. Vasospasms can be prevented using slow injection times ${ }^{33}$ and hence the recommended injection rate of Onyx ${ }^{\circledR}$ is $0.16 \mathrm{~mL} \mathrm{~min}{ }^{-1}$. ${ }^{24}$ The administration of Onyx ${ }^{\mathbb{R}}$ is requires use of specialist syringes and microcatheters due to degradation of the more commonly employed versions by the DMSO solvent. ${ }^{32}$ Suitable microcatheters for injection of the DMSO based liquid embolic include Rebar ${ }^{\mathrm{TM}}$ (Medtronic Inc.), Progreat ${ }^{\mathbb{R}}$ (Terumo) and Apollo ${ }^{\mathrm{TM}}$ (Medtronic Inc.).

This precipitating system resolves some of the issues experienced with $n$ BCA glue as Onyx ${ }^{\mathbb{R}}$ is non-adhesive in nature and so has a reduced risk of microcatheter blockage and entrapment. ${ }^{34,35}$ The entrapment is further reduced with the use of the manufacturers (Medtronic Inc.) specialist DMSOcompatible microcatheter, Apollo ${ }^{\mathrm{TM}}$, with a detachable tip which can be left in place inside the patient if the microcatheter tip becomes entrapped in the solidified embolic plug during the procedure. This provides a safe and effective method of dealing with microcatheter entrapment if it occurs to reduce patient risk of complication associated with leaving a large length of microcatheter in place. The delivery process can also be carried out using intermittent injections allowing the delivery to be paused to evaluate the progress of the embolus and then continued with further penetration of the embolus into the vasculature. As the solution is injected through the catheter, a small mass of polymer gel forms and adheres to the tip, swelling as further solution is administered. A skin forms at the solution-blood interface as the material precipitates, forming a solution filled sac which is further expanded by the growing mass of fresh solution exiting the catheter tip. This causes continuous rupture of the forming skin as the embolus continues to expand with subsequent reskinning as the freshly exposed solution precipitates. This process has often been described as 'lava-like' in nature and allows for level of control over the procedure not possible with glue-based embolics. ${ }^{36}$ This 'pushability' of the material in paused increments means a single microcatheter can be used for multiple injections during each procedure without the need to place new microcatheters after each injection. Once sufficient material has been delivered, the end-point of the embolisation is determined when the delivered liquid embolic no longer flows within the vessel due to its solidification and is easily visualised under real-time fluoroscopy. Following implantation of Onyx ${ }^{\mathbb{R}}$, the solidified embolic material can be readily located by X-ray imaging techniques. 


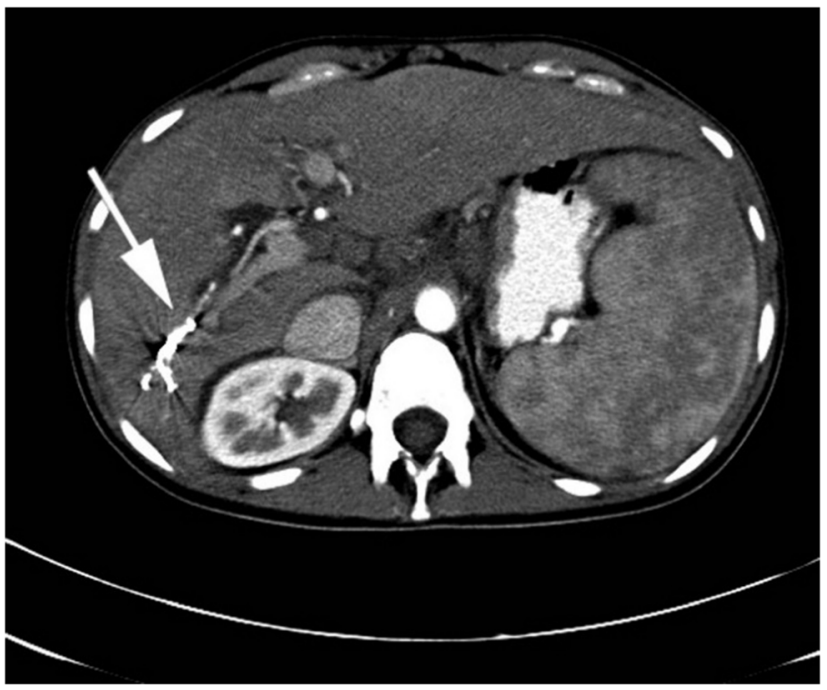

Fig. 5 Beam hardening and streak artefact (indicated by white arrow) observed with Onyx ${ }^{\mathbb{R}}$ in the right hepatic artery by contrast enhanced axial CT. Reproduced from Clin. Radiol., 2015, 70, 326-32. ${ }^{37}$

Using computed tomography (CT) imaging, which is conventionally used for patient follow-up, the embolic is visualised as a dense bright white region due to the high radiopacity imparted by the tantalum radiopacifying agent. However, due to the metallic nature and high radiodensity of tantalum, Onyx ${ }^{\circledR}$ often exhibits streak artefacts under X-ray imaging as a result of beam hardening and scattering of the X-ray energy used during scanning (Fig. 5). ${ }^{37}$ This imaging artefact can obscure the surrounding anatomy of the treated vascular abnormalities and hinder further diagnosis or monitoring of disease progression. This can also pose difficulties in assessment of optimal embolisation or requirement of further treatments. For this reason, Onyx manufacturers Ev3 recently launched a new "L" range of Onyx which contains less tantalum to reduce the incidence of imaging artefacts.

\subsection{Lipiodol $^{\circledR}$}

Lipiodol Ultrafluide ${ }^{\circledR}$ (Guerbet Llc.) is a contrast agent based on a mixture of ethyl esters of iodinated poppy seed oil that was invented in 1901 and is indicated for use in lympangiography. It has been used off label for many years to create viscous emulsions of chemotherapy for treatment of hepatocellular carcinoma, whereby the high viscosity causes reduction of blood flow in the tumour and minimises drug wash-out and hence could be classed as a transient liquid embolic agent. The ethiodised oil is often mixed with the anticancer agent doxorubicin or combinations of multiple chemotherapeutic agents, ${ }^{38}$ followed by a subsequent embolisation of the feeding tumour vessels performed using particles or microspheres in order to permanently block the blood flow; this procedure is termed conventional Trans-Arterial Chemo-Embolisation (cTACE). Embolisation by combination of Lipiodol ${ }^{\mathbb{R}}$ with other embolic devices has been demonstrated to improve tumour response rates to treatment versus just Lipiodol emulsion alone. ${ }^{39}$

The contrast agent provides a temporary embolic effect in larger arteries ${ }^{40}$ but ultimately accumulates in hypervascularised tumours as there is no mechanism for its removal due to the lack of Kuppfer cells in tumour tissue. ${ }^{41}$ The retention of Lipiodol ${ }^{\mathbb{R}}$ within tumours is readily imaged under X-ray based methods and is beneficial to the physician as it provides an indication that the tumour has been successfully targeted. ${ }^{5}$ The treatment of HCC by cTACE is performed in a set of at least two sequential cTACE procedures with significant response often observed as tumour necrosis by the second treatment. ${ }^{42}$

\section{Emerging liquid embolics}

Potential materials for use as liquid embolics can be spilt into 3 main types; polymerising, precipitating and phase transitioning. Polymerising systems contain monomers or macro-monomers in a carrier solution which polymerise upon contact with a suitable initiator. The initiator can be mixed prior to the delivery of the polymerising embolic allowing these materials to gel over a predictable and predetermined time. Therefore, when using polymerising systems premixed with an initiator, delivery must be carried out within a limited time-frame in order to provide effective embolisation and avoid microcatheter blocking and entrapment. An alternative to this is to deliver polymerising system via a dual-lumen microcatheter to ensure the reacting components are kept separate during delivery until mixing at the tip-section of the microcatheter. This delivery method relies on a rapid rate of reaction to prevent wash-out of the embolic material by the blood flow before gelation has occurred and specialist catheter construction which can be expensive and may hinder the trackability of the catheter, limiting its ability to navigate close to the target site.

Precipitating systems are administered as a polymer dissolved in a low toxicity and biocompatible carrier solvent. On contact with a precipitation stimulant, such as non-solvent of aqueous conditions or salt present within the blood, precipitation of the previously dissolved polymer chains occurs to form a gel in situ. Following gelation, the carrier solvent diffuses away and generally aqueous-based media from the blood replaces it within the polymer matrix. Precipitating polymers offer the advantage that they form gels only when in contact with precipitating stimulus present within physiological fluids and therefore avoid the problem of gelation within the microcatheter which poses the risk of microcatheter blockage. This allows delivery to be paused during the embolisation procedure in order to prevent escape of material into non-target vessels and allow more control. There are some disadvantages associated with precipitating liquid embolics in that if the onset of precipitation is not fast enough, the blood flow through the vessel can result in wash-out leading to local or systemic toxicity as a result of the free polymer. Also, a suitable carrier solvent must be selected in order to avoid any toxic effects on injection into the vasculature.

In addition to polymerising and precipitating systems, polymers which undergo a sol-gel phase transition in response to external stimuli have also been demonstrated to be useful in the area of therapeutic embolisation. These materials respond 
to their surrounding environment due to changes in parameters such as temperature, $\mathrm{pH}$, ion concentrations or electric fields. Hence, phase transitioning polymers can be selected which respond to physiological parameters such as body temperature, blood $\mathrm{pH}$ or the salt concentrations in blood. Phase transitioning systems can be designed to gel under conditions similar to those experienced in an embolisation procedure such as the change in temperature of the injected liquid from room temperature to body temperature as its injected through the microcatheter or the contact with blood and the change in $\mathrm{pH}$ and ionic strength as the liquid exits the microcatheter at the delivery site. There is a limitation for phase transitioning materials that are solely thermo-responsive and gel at temperatures around that of body temperature. This is due to the long lengths of microcatheters used (typically $100-150 \mathrm{~cm}$ in length) which can culminate in the injected liquid solution reaching body temperature as it travels along the length of the microcatheter. This will likely result in microcatheter blockage if the phase transition occurs before exiting the microcatheter.

\subsection{Polymerising formulations}

3.1.1 Instylla hydrogel embolic system. Instylla's Hydrogel Embolic System (Instylla - Incept Inc.) is a polyethylene glycolbased system which polymerises in situ on mixing with an initiator. The two components are injected from separate syringes and flow down a dual lumen arrangement in the microcatheter, formed by inserting a narrow inner tube within the microcather lumen that finishes short of the microcatheter tip to allow a short mixing zone. The low viscosity liquid undergoes rapid gelation to form an embolic gel within the vasculature. The polymeric material is not radiopaque, hence the material must be mixed with contrast agent prior to delivery to monitor the injection.

The product has been tested in animal models in which high vascular penetration was observed likely due to the low viscosity of the liquid embolic. The liquid embolic system has been tested using a range of microcatheters, including balloon microcatheters, and alongside coil embolisation treatments. Successful polymerisation of the gel was also observed in high

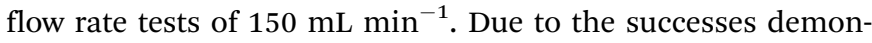
strated in early testing of the liquid embolic system, further in vitro and in vivo testing of Instylla's Hydrogel Embolic System is currently being performed. ${ }^{43}$

3.1.2 PPODA-QT. Vernon et al reported on a polymerising system used in the filling of aneurysms in a swine model which is composed of poly(propylene glycol)diacrylate (PPODA) that is mixed with pentaerythritol tetrakis(3-mercaptopropionate) (QT). ${ }^{44}$ The gelling solution is formulated with a catalytic amount of hydroxide $\left(\mathrm{HO}^{-}\right)$that deprotonates the QT thiol group which then undertakes nucleophilic attack of the PPODA acrylate group in a Michael-type addition reaction (Fig. 6). The rate of gelation can be controlled by changing the $\mathrm{pH}$ but injection over a 1-2 minute timeframe was reported to take around 10 minutes.

\subsection{Precipitating formulations}

3.2.1 Squid $^{\mathrm{TM}} /$ Squid Peri ${ }^{\mathrm{TM}}$. Squid ${ }^{\mathrm{TM}}$ and Squid Peri ${ }^{\mathrm{TM}}$ (Emboflu, Switzerland) are similar to Onyx ${ }^{\circledR}$ in that they are precipitating liquid embolics composed of an EVA copolymer in a DMSO suspension with micronised tantalum powder. Squid ${ }^{\mathrm{TM}}$ is indicated for neuro applications and Squid Peri ${ }^{\mathrm{TM}}$ for peripheral use. In both Squid $^{\mathrm{TM}}$ formulations a smaller grain size of tantalum powder is used which has a slower rate of sedimentation in comparison to Onyx ${ }^{\circledR}$ (Fig. 7). ${ }^{45}$ This provides longer working times of the material due to its ability to retain homogeneous suspension over a longer time frame which is particularly useful when using prolonged injection times. Squid ${ }^{\mathrm{TM}}$ is marketed in a range of formulations including Squid ${ }^{\mathrm{TM}} 12$ and Squid $^{\mathrm{TM}} 18$ which have viscosities of $12 \mathrm{mPa}$ s and $18 \mathrm{mPa}$, respectively. This allows the appropriate viscosity to be selected depending on the desired clinical outcome, for example the lower viscosity Squid ${ }^{\mathrm{TM}} 12$ is ideal for achieving deeper penetration into the vasculature often required with the treatment of AVMs. Whereas, Squid ${ }^{\mathrm{TM}} 18$ is better
A - PPODA<smiles>C=CC(=O)OCC(C)OC(C)C=C</smiles>

C - Michael-Type Addition
B - QT<smiles>O=C(CCS)CCC(COC(=O)CCS)(COC(=O)CCS)COC(=O)CCS</smiles><smiles>[R]SCCC(=O)OCC(C)C</smiles>

A Poly-(propylene glycol)-diacrylate (PPODA)

B Pentaerythritol tetrakis-(3-mercaptopropionate) (QT)

C Michael-Type Addition - Nucleophilic thiol group of QT adds to acrylate of PPODA under basic conditions

Fig. 6 Components \& reaction scheme for gelation of PPODA-QT 

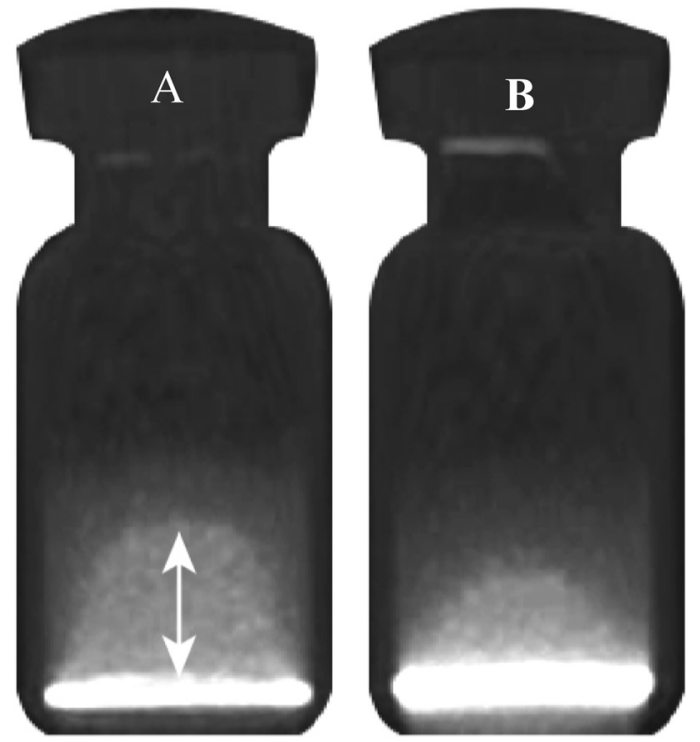

Fig. 7 X-ray images showing the longer tantalum suspension time (as indicated by the white arrow) of Squid ${ }^{\mathrm{TM}} 18(\mathrm{~A})$ in comparison to Onyx ${ }^{\mathbb{R}} 18$ (B) 15 minutes after agitation (image from Emboflu promotional brochure for Squid $\left.{ }^{\mathrm{TM}}\right)$.

suited to the treatment of endoleaks where the higher viscosity of Squid $^{\mathrm{TM}} 18$ is better able to resist wash out by blood flow. Squid $^{\mathrm{TM}}$ formulations are also available with $30 \%$ less tantalum in the suspension, marketed as Squid $^{\mathrm{TM}} 12 \mathrm{LD}$ and Squid $^{\mathrm{TM}}$ 18LD. ${ }^{46}$ The lower amount of tantalum in the liquid embolic formulation reduces the imaging artefact of streaking which is often observed with Onyx ${ }^{\circledR}$. Despite the improved properties over the well-established Onyx ${ }^{\mathbb{R}}$ treatment, Squid ${ }^{\mathrm{TM}}$ has not found widespread use in clinic likely due to its high cost and requirement of DMSO compatible microcatheters.

3.2.2 Precipitating hydrophobic injectable liquid $\left(\mathrm{PHIL}^{\circledR}\right)$. The precipitating hydrophobic injectable liquid PHIL ${ }^{\circledR}$ (MicroVention (Terumo Corp)) is indicated for the embolisation of the peripheral and neurovasculature, including AVMs and hypervascular tumours. ${ }^{47-49}$ The liquid embolic is delivered using DMSO as the carrier solvent which on mixing with blood diffuses away as the polymeric material precipitates out of solution on contact with the aqueous environment within the vasculature to form a solid embolus. The polymer is based on a 2,4,6-triidophenol-lactide-lactide-co-glycolic acrylate and hydroxyethyl-methacrylate (HEMA) (2:1 monomer ratio) copolymer which is non-adhesive in nature so avoids adhesion to the microcatheter during delivery (Fig. 8). Again, due to the DMSO carrier solvent used in the formulation, PHIL ${ }^{\circledR}$ must be used with specialist DMSO compatible connectors and microcatheters, similar to embolisation using Onyx ${ }^{\circledR}$ or Squid ${ }^{\mathrm{TM}}$. The radiopacifying agent iodine is covalently bound to the polymer, hence no pre-mixing of materials is required to achieve a homogeneous suspension before delivery as is the case for tantalum based radiopacifying agents and again, less streak artefacts in imaging has been noted..$^{50}$ Therefore, PHIL ${ }^{\circledR}$ is supplied in pre-loaded syringes which reduces the overall procedural time. It is also marketed in a range of viscosities $(25,30$ and $35 \mathrm{wt} \%$ concentrations giving 16,36 and $72 \mathrm{mPa} \mathrm{s}$ solution viscosities) allowing the appropriate viscosity to be selected for individual treatments depending on the desired level of vascular penetration and flow rate scenarios.

It has been demonstrated that $\mathrm{PHIL}^{\circledR}$ precipitates with a greater volume of precipitate formed per $\mathrm{mL}$ of liquid embolic injection than Onyx ${ }^{\mathbb{R}},{ }^{52}$ thereby requiring a reduced volume of liquid embolic in the embolisation process potentially reducing materials cost. In a study of AVM treatment followed by resection, no major microcatheter blockages were reported during use and histological analysis of the PHIL material in the tissues showed it to be quite different to Onyx, being more brittle and less pliable in nature and eliciting a greater inflammatory reaction in the surrounding tissue. ${ }^{50}$

3.2.3 Iodinated polyvinyl alcohol (I-PVAL). A precipitating liquid embolic based upon iodinated polyvinyl alcohol (I-PVAL) dissolved in $\mathrm{N}$-methyl pyrrolidone (NMP) was reported in the literature some years ago and evaluated in a porcine model of wide-necked aneurysms. ${ }^{53}$ The material proved safe and effective with good visibility but only more recently has it been commercialised by Antia Therapeutics AG. This precipitating liquid embolic known as Easyx ${ }^{\mathrm{TM}}$, is still composed of PVAL (also known as PVA or PVOH) with iodine containing moieties covalently bound to the polymer backbone through ether<smiles>[Y4]C(OC(=O)COC(=O)C(C)(CC(C)C)CC(C)C(=O)OC(C)C(=O)OC(C)C(=O)Oc1c(I)cc(I)cc1I)C(=O)OC(C)C(=O)Oc1c(I)cc(I)cc1I</smiles>

Fig. 8 Chemical structure of the PHIL copolymer - The arrangement of glycolide/lactide/lactide units in the iodinated monomer is likely randomised on the PHIL product. Ratio Monomer : HEMA is estimated to be $2: 1^{51}$ 
linkages (Fig. 9). ${ }^{54}$ However now, as with Onyx ${ }^{\circledR}$, Squid $^{\mathrm{TM}}$ and PHIL $^{\circledR}$, the product is formulated in DMSO as the carrier solvent which diffuses away from the embolus as the polymer precipitates out of solution on contact with the aqueous conditions of blood. The exact reason for the developers switching carrier solvent is unknown but may be as a result of a simpler regulatory pathway to product approval. As with the other products, this requires the use of DMSO compatible microcatheters to prevent the deterioration of the microcatheters by the solvent. The inherent radiopacity imparted by the covalently bound iodine allows the liquid embolic to be clearly visualised by computed tomography (CT) imaging without the shading and beam-hardening artefacts observed with the tantalum radiopacifying agent used with Onyx ${ }^{\circledR} .55$ Also, the covalent binding of the radiopaque group alleviates the need to pre-mix the liquid embolic with a radiopacifying agent prior to delivery as the radiopaque materials are supplied in a ready to use solution in DMSO. Easyx ${ }^{\mathrm{TM}}$ has been developed for the embolisation of hypervascular lesions including tumours and AVMs. The liquid embolic is currently undergoing clinical trials but early data has demonstrated effective embolisation with homogeneous visibility under CT imaging. ${ }^{55-57}$

3.2.4 Eudragit-E (poly(MM-co-BM-co-DMAEMA)). Tamura et al. reported on the endovascular embolisation of brain AVMs using a copolymer of methyl methacrylate, butyl methacrylate and dimethylaminoethyl methacrylate (poly(MM-co-BM-coDMAEMA), Fig. 10) known as Eudragit-E and dissolved in a carrier mixture of $50: 50$ ethanol and iopamidol contrast agent. ${ }^{58}$ The polymer itself has a history of medical use as a tablet coating for taste masking and is one of a family of amorphous nonbiodegradable, non-absorbable, non-toxic materials marketed under the generic Eudragit name (Evonik Industries, Germany). Prior to delivery, the microcatheter needs to be first flushed with ethanol to prevent premature precipitation and occlusion of the lumen. Injection into the swine Rete mirabilia (a common model for AVM evaluation) and subsequent histopathology has revealed a tissue reaction similar to $n \mathrm{BCA}$ with inflammation and thrombosis accompanied by extensive endothelial damage. ${ }^{59}$ This is likely to be largely due to the toxicity of the ethanol carrier, which is itself a sclerosant. There was not however, any evidence of haemorrhage or extravasation of the material. Radiopacity is only transient, provided by the iopamidol contrast agent which

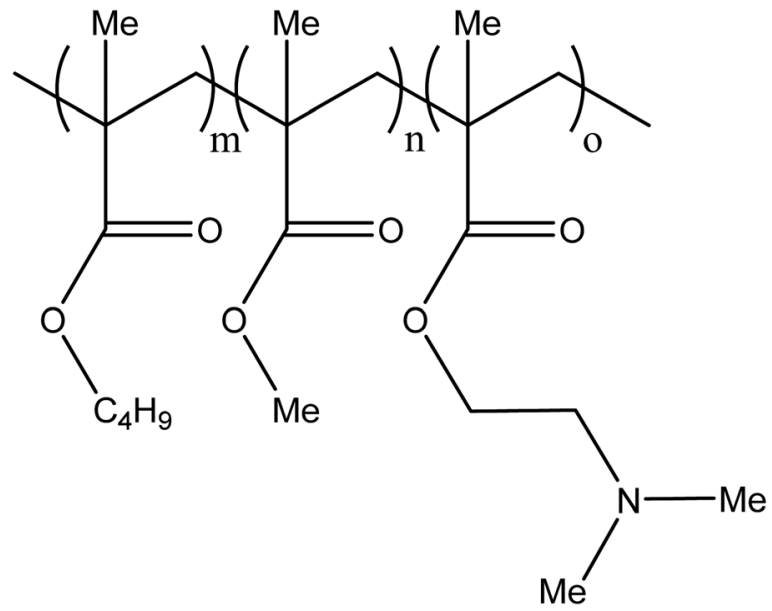

Fig. 10 Chemical structure of Eudragit E.

eventually diffuses out of the precipitated polymer mass. Despite these promising findings, there is little in the literature since these reports to suggest the company is actively developing this as a liquid embolic commercial offering.

\subsection{Phase transitioning}

3.3.1 GPX. GPX (Fluidx Medical Technology) is a biomimetic aqueous based liquid embolic system. The liquid embolic is based on the liquid glue secreted by sandcastle worms to create tubular structures using grains of sand. ${ }^{60,61}$ Using the same principal as the sandcastle worms, GPX consists of polyelectrolyte complexes which are stabilised in aqueous solutions of high ionic strength (Fig. 11). The formulation consists of protamine sulfate (from salmon sperm) and sodium inositol hexaphosphate (phytic acid), is rendered radiopaque by addition of tantalum powder particles $(1.5 \mu \mathrm{m}$ in size $)$ and maintained in an un-coacervated state by the addition of $1.2 \mathrm{M}$ sodium chloride. The stabilised solution can be delivered through the narrow lumens of microcatheters used in embolisation procedures. On exiting the microcatheter at the injection site, the polyelectrolyte complexes become destabilised due to the reduction of the ionic strength to physiological strength through blood dilution. This causes the polyelectrolyte complexes to aggregate to form a gelled embolus within the
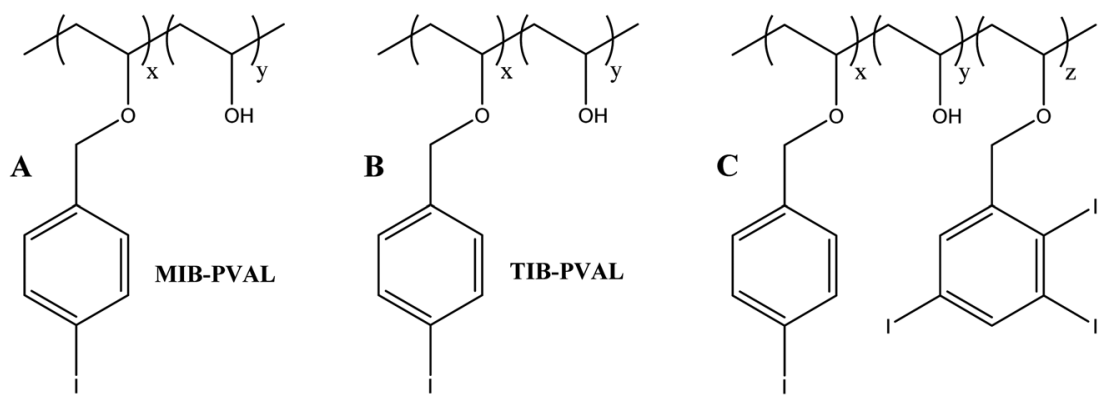

Fig. 9 Putative structure of Easyx ${ }^{\mathrm{TM}}$ copolymer - The product is likely be either a mixture of poly(vinyl-4-mono-iodo benzyl ether) MIB-PVAL (A) \& poly(vinyl-2,3,5-triiodo-benzyl ether) TIB-PVAL (B), or copolymer C. 

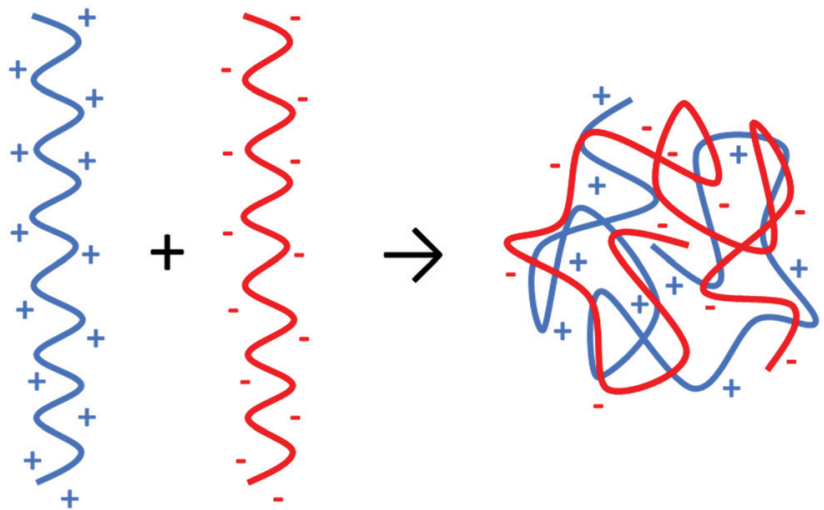

Fig. 11 Schematic of the complexation of a polyelectrolyte from a polyanion and polycation.

vasculature with rapid gelation exhibited within seconds. Two different viscosity formulation are being proposed which have a toothpaste-like delivery and control. The resulting embolic mass is non-cytotoxic, non-haemolytic, and non-inflammatory. Despite the fact the material is based upon a glue-like substance, the embolus formed was also found to be non-adhesive in nature to the microcatheter used to deliver the embolic material. Embolisation using GPX has been performed in rabbit renal arteries demonstrating a high level of vascular penetration and complete occlusion of the kidney. ${ }^{62}$

3.3.2 Calcium alginate. Calcium alginate is a widely used material in medical applications ${ }^{63}$ and its use as a liquid embolic agent was reported some years ago.$^{64}$ Alginate is a copolymer composed of units of mannuronic and guluronic acid groups that are ionically crosslinked by divalent cations such as $\mathrm{Ca}^{2+}$ (Fig. 12). A concentric tube microcatheter design was used in the evaluation of this system in an animal model of side-wall aneurysms. Delivery was feasible with no off-target fragmentation and analysis at 30 and 90 days showed the alginate allowed for tissue overgrowth of the aneurysm opening with moderate fibrous tissue formation. ${ }^{64}$ In a later study by
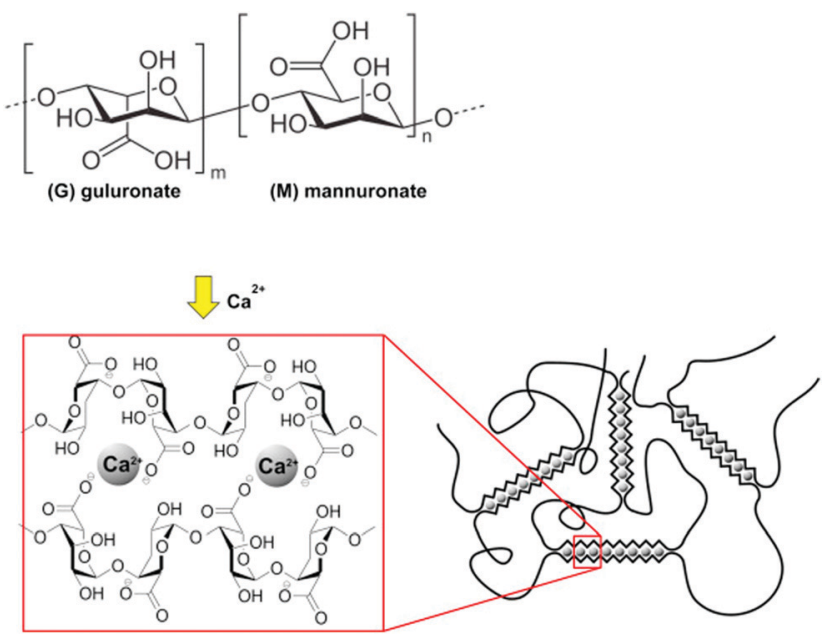

Fig. 12 Structure and crosslinking mechanism for sodium alginate. Reproduced from Carbohydr. Polym., 2015, 131, 57-64. ${ }^{67}$ other investigators, co-delivery of alginate plus iohexol contrast agent in one stream and calcium ions in the other, with mixing at the exit point of the microcatheter, resulted in a string-like gel (termed Embogel). ${ }^{65}$ This was shown to effectively fill fusiform and saccular aneurysm models when used in conjunction with a AAA stent. The need for specialist microcatheters with reduced trackability limits the locations that can be targeted and adds cost to the procedure and hence has hindered the continued development of alginate-based systems. This has led researchers to attempt to modify the properties by ad-mixing with other components and in one report alginate, calcium, Poloxamer 407, hydroxymethylcellulose and iodixanol to add radiopacity were formulated in order to produce a liquid embolic composition that had thermosensitive properties, transitioning between flowing sol to gel at body temperature ${ }^{66}$ and proved feasible to deliver and embolise rabbit renal arteries.

3.3.3 Temperature sensitive hydrogel systems. A phase transitioning injectable material has been developed using a Silk Elastin Protein Polymer (SELP) (Fig. 13). Phase transition of the polymeric material occurs in response to temperature undergoing a sol-gel transition on an increase in temperature from room temperature to body temperature at $37^{\circ} \mathrm{C} .{ }^{68}$ Feasibility of the material as an injectable material has been demonstrated using an in vitro and in vivo model. ${ }^{69,70}$ The product is currently under further investigation for use as a therapeutic gene delivery system.

A temperature sensitive poly( $N$-isopropylacrylamide-co-butyl methacrylate) (PNIPAM-BM, or PIB) nanogel-forming material has also been described for use as a liquid embolic agent, ${ }^{71}$ based upon the widely known phase transition of PNIPAM from hydrophilic to hydrophobic in nature above its lower critical solution temperature (LCST). This system has the advantage of not requiring an organic solvent being aqueous-based and is forms a non-adhesive hydrogel that does not stick to the catheter. It has no intrinsic radiopacity and required mixing with iohexol contrast agent in order to impart temporary radiopacity. Rapid delivery $\left(>0.1 \mathrm{~mL} \mathrm{~s}^{-1}\right)$ was required to ensure distribution to the more peripheral vessels before gelation occurred and indeed, slow delivery resulted in proximal occlusion with the risk of microcatheter blockage if the injection was stopped making the procedure impractical.

A material based upon chitosan/ $\beta$-glycerophosphate has been described, with similar properties to PIB in that it phasetransitions at room temperature to form a non-adhesive,

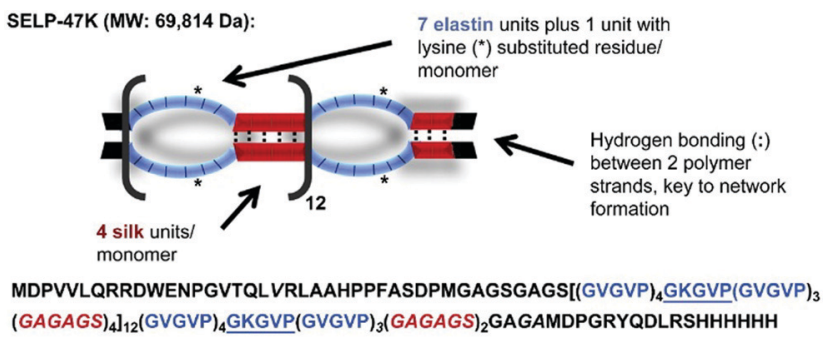

Fig. 13 Amino acid composition of a silk elastin protein polymer (SELP-47 K). Image adapted from Biomater., 2015, 57, 142-52. 
A

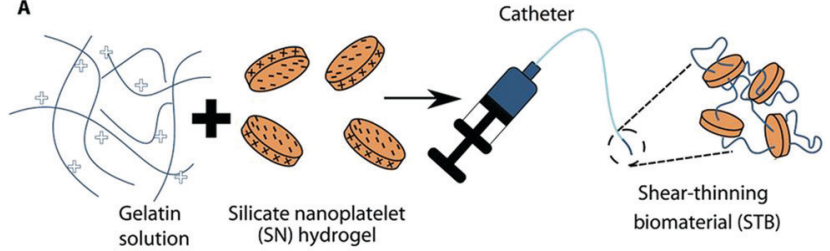

Fig. 14 Diagram of shear thinning behaviour of a nanocomposite hydrogel containing gelatin and silicate nanoplatelets. Image adapted from Sci. Transl. Med., 2016, 8, 365-77.

non-toxic hydrogel at $37{ }^{\circ} \mathrm{C}$. This material was evaluated in a rabbit renal artery model ${ }^{72}$ and in the rete mirabile in swine. ${ }^{73}$ Interestingly, these materials have been formulated with indocyanine green and evaluated for potential intraoperative fluorescent imaging and local therapy of hepatocellular carcinoma. ${ }^{74}$ Whilst the dye did not affect the gelation rate, the gel transition temperature was above $4{ }^{\circ} \mathrm{C}$ making the handling and avoidance of premature gelling of this material very difficult.

3.3.4 PuraMatrix ${ }^{\mathrm{TM}}$. PuraMatrix ${ }^{\mathrm{TM}}$ (3D Matrix Co., Ltd, Tokyon, Japan) is based upon a peptide with 16 amino acids comprised of arginine, aspartate and alanine repeating units (RADARADARADARADA, RADA16-I). Although originally developed as a cell scaffold material, it has been formulated at $2.5 \mathrm{wt} \%$ in aqueous media mixed with iodinated contrast agent to impart radiopacity as a proposed liquid embolic agent. ${ }^{75}$ This peptide sequence has an alternating hydrophobic/charged motif that promotes $\beta$-strand formation and self-assembly into a nanofiber-based hydrogel material. ${ }^{76}$ Evaluation in an embolisation setting confirmed the ability of the material to gel and induce cessation of blood flow in target vessels. Pathological analysis revealed an ability to reach distal locations due to its non-adhesive nature and little inflammation in the vessel and surrounding tissues.

3.3.5 Shear-thinning biomaterials. Shear-thinning biomaterials (STB) are an attractive approach to liquid embolics as their rheological properties are such that their viscosity drops as it is sheared by injection through the narrow lumen of a microcatheter. Once the shear stress is removed as it exits the catheter and enters the less confined environment of the blood vessel, the material returns to its gel state and form an embolus in situ. Currently under investigation as a liquid embolic alternative is a STB based on a nanocomposite hydrogel composed of gelatin and silicate nanoplatelets (Fig. 14). ${ }^{70,77}$ This STB demonstrated injectability through a range of microcatheters and needles and formed a sufficient embolus that could withstand physiological pressures without displacement in the vasculature. The application of shear disrupts charge interactions between the gelatin and silicate particles, dropping the viscosity as the apparent molecular weight decreases, which are restored again to reinforce the gel once the shear is removed.

The aqueous nature of the system alleviates the need to use DMSO compatible microcatheters as required by a number of liquid embolics. However, the material used in the GPX formulation is not inherently radiopaque so would need to be delivered alongside a contrast agent to enable visualisation of the embolisation procedure. Liquid contrast agent would likely alter the rate of gelation of the liquid embolic system and particulate tantalum may interfere with the gelatin-silicate interactions, hence further testing of the STB is still required.

\section{Conclusions and future perspectives}

There are numerous liquid embolics currently under development aiming to alleviate some of the current issues faced with commercially available liquid embolics and provide additional tools for use in therapeutic embolisation. There are however, a number of prerequisites which must be fulfilled in order to demonstrate improved properties and clinical benefit over the currently established agents already available on the market. These properties include reduced streak artefact on imaging, appropriate gelation triggers to avoid catheter blockage and suitable viscosities to occlude at the desired location under different flow regimes within the vasculature and control over the delivery without risk of fragmentation and stringing to offtarget sites. Some desirable attributes for the next generation of liquid embolics would be the possession of inherent radiopacity to avoid the need to pre-mix materials prior to injection and systems which are free of solvent to reduce the risk of toxic side effects often associated with the use of solvent and the need to use solvent-compatible microcatheters and delivery accessories. These properties are currently unmet needs and the next generation of liquid embolics systems reviewed herein are likely to address these to a greater or lesser extent.

\section{Conflicts of interest}

ALL and HB are employees of Biocompatibles UK Ltd, a developer of embolisation products for use in minimally invasive procedures. JL and SGS have received funding from Biocompatibles UK Ltd.

\section{Acknowledgements}

J. L. would like to thank Biocompatibles UK Ltd and the UK EPSRC Centre for Doctoral Training in Polymers \& Soft Matter (EP/L016281/1) for funding a CASE PhD studentship.

\section{References}

1 J. D. Barr, T. J. Lemley and C. N. Petrochko, J. Vasc. Interv. Radiol., 1998, 9, 113-118.

2 R. Duran, K. Sharma, M. R. Dreher, K. Ashrafi, S. Mirpour, M. Lin, R. E. Schernthaner, T. R. Schlachter, V. Tacher, A. L. Lewis, S. Willis, M. den Hartog, A. Radaelli, A. H. Negussie, B. J. Wood and J.-F. H. Geschwind, Theranostics, 2016, 6, 28-39.

3 T. Yonemitsu, N. Kawai, M. Sato, T. Sonomura, I. Takasaka, M. Nakai, H. Minamiguchi, S. Sahara, Y. Iwasaki, T. Naka 
and M. Shinozaki, Cardiovasc. Intervent. Radiol., 2010, 33, 1192-1197.

4 T. Yonemitsu, N. Kawai, M. Sato, H. Tanihata, I. Takasaka, M. Nakai, H. Minamiguchi, S. Sahara, Y. Iwasaki, Y. Shima, M. Shinozaki, T. Naka and M. Shinozaki, J. Vasc. Interv. Radiol., 2009, 20, 1176-1187.

5 R. Lencioni, Hepatology, 2010, 52, 762-773.

6 L. Pierot, C. Cognard, D. Herbreteau, H. Fransen, W. J. van Rooij, E. Boccardi, A. Beltramello, N. Sourour, K. Kupcs, A. Biondi, A. Bonafé, W. Reith and A. Casasco, Eur. Radiol., 2013, 23, 2838-2845.

7 E. J. Speir, R. M. Ermentrout and J. G. Martin, Tech. Vasc. Interv. Radiol., 2017, 20, 258-262.

8 A. J. Ringer and R. Rahme, in Cerebrovascular and Endovascular Neurosurgery, Springer International Publishing, Cham, 2018, pp. 321-333.

9 F. Leonard, R. K. Kulkarni, G. Brandes, J. Nelson and J. J. Cameron, J. Appl. Polym. Sci., 1966, 10, 259-272.

10 H. V. Vinters, K. A. Galil, M. J. Lundie and J. C. E. Kaufmann, Neuroradiology, 1985, 27, 279-291.

11 R. J. Rosen and S. Contractor, Semin. Intervent. Radiol., 2004, 21, 59-66.

12 M. F. Brothers, J. C. Kaufmann, A. J. Fox and J. P. Deveikis, Am. J. Neuroradiol., 1989, 10, 777-786.

13 R. I. White, J. V. Strandberg, G. S. Gross, K. H. Barth, T. F. Groves and F. Starr, Radiology, 1977, 125, 677-687.

14 G. Wikholm, Am. J. Neuroradiol., 1995, 16, 479-482.

15 S. Vaidya, K. R. Tozer and J. Chen, Semin. Intervent. Radiol., 2008, 25, 204-215.

16 M. J. Gounis, B. B. Lieber, A. K. Wakhloo, R. Siekmann and L. N. Hopkins, Am. J. Neuroradiol., 2002, 23, 938-944.

17 H. Hill, J. F. B. Chick, A. Hage and R. N. Srinivasa, Diagn. Interv. Radiol., 2018, 24, 98-103.

18 Y. Takeuchi, H. Morishita, Y. Sato, S. Hamaguchi, N. Sakamoto, H. Tokue, T. Yonemitsu, K. Murakami, H. Fujiwara, K. Sofue, T. Abe, H. Higashihara, Y. Nakajima and M. Sato, Jpn. J. Radiol., 2014, 32, 500-517.

19 J. S. Pollak and R. I. White, J. Vasc. Interv. Radiol., 2001, 12, 907-913.

20 The $n$-BCA Trial Investigators, Am. J. Neuroradiol, 2002, 23, 748-755.

21 J. Mathis, A. Evans, A. DeNardo, K. Kennett, J. Crandall, M. Jensen and J. Dion, Am. J. Neuroradiol, 1997, 18, 1087-1091.

22 B. C. Flores, A. P. See, G. M. Weiner, B. T. Jankowitz, A. F. Ducruet and F. C. Albuquerque, J. Neurosurg., 2019, 130, 963-971.

23 S. Paramasivam, D. Altschul, S. Ortega-Gutiarrez, J. Fifi and A. Berenstein, J. Neurointerv. Surg., 2015, 7, 485-461.

24 Micro Therapeutics, Onyx Liquid Embolic System - Instructions for Use, 2003.

25 S. Luzzi, M. Del Maestro, D. Bongetta, C. Zoia, A. V. Giordano, D. Trovarelli, S. Raysi Dehcordi and R. J. Galzio, World Neurosurg., 2018, 116, 340-353.

26 W. J. van Rooij, M. Sluzewski and G. N. Beute, Am. J. Neuroradiol., 2007, 28, 172-177.
27 D. Raissi, Q. Yu and S. H. Mardini, J. Clin. Imaging Sci., 2018, 8, 46.

28 N. P. Munro, S. Woodhams, J. D. Nawrocki, M. S. Fletcher and P. J. Thomas, BJU Int., 2003, 92, 240-244.

29 R. Siekmann, Interv. Neuroradiol., 2005, 11, 131-140.

30 R. Ashour and M. Ali Aziz-Sultan, Neurol. Res., 2014, 36, 363-367.

31 R. Loffroy, S. Favelier, P.-Y. Genson and B. Guiu, Cardiovasc. Intervent. Radiol., 2012, 35, 221.

32 J. C. Chaloupka, F. Vinuela, H. V. Vinters and J. Robert, Am. J. Neuroradiol, 1994, 15, 1107-1115.

33 J. C. Chaloupka, D. C. Huddle, J. Alderman, S. Fink, R. Hammond and H. V. Vinters, Am. J. Neuroradiol, 1999, 20, 401-410.

34 W. Taki, Y. Yonekawa, H. Iwata, A. Uno, K. Yamashita and H. Amemiya, Am. J. Neuroradiol, 1990, 11, 163-168.

35 T. Terada, Y. Nakamura, K. Nakai, M. Tsuura, T. Nishiguchi, S. Hayashi, T. Kido, W. Taki, H. Iwata and N. Komai, J. Neurosurg., 1991, 75, 655-660.

36 R. Regine, F. Palmieri, M. De Siero, A. Rescigno, V. Sica, R. Cantarela and V. Villari, Interv. Med. Appl. Sci., 2015, 7, 22-29.

37 J. B. Jia, C. S. Green, A. J. Cohen and M. Helmy, Clin. Radiol., 2015, 70, 326-332.

38 J. M. Llovet, M. I. Real, X. Montaña, R. Planas, S. Coll, J. Aponte, C. Ayuso, M. Sala, J. Muchart, R. Solà, J. Rodés and J. Bruix, Lancet, 2002, 359, 1734-1739.

39 K. Takayasu, Y. Shima, Y. Muramatsu, N. Moriyama, T. Yamada, M. Makuuchi, H. Hasegawa and S. Hirohashi, Radiology, 1987, 163, 345-351.

40 T. de Baère, A. Denys, R. Briquet, P. Chevallier, J. Dufaux and A. Roche, J. Vasc. Interv. Radiol., 1998, 9, 305-310.

41 T. de Baere, X. Zhang, B. Aubert, G. Harry, C. Lagrange, J. Ropers, J. Dufaux, J. Lumbroso, P. Rougier, M. Ducreux and A. Roche, Radiology, 1996, 201, 731-735.

42 C. Georgiades, J. F. Geschwind, N. Harrison, A. HinesPeralta, E. Liapi, K. Hong, Z. Wu, I. Kamel and C. Frangakis, Radiology, 2012, 265, 115-123.

43 S. Ganguli, J. Weintraub, T. DiBartholomeo, R. Lareau, H. Claesson and R. Bean, J. Vasc. Interv. Radiol., 2019, 30, S170.

44 C. R. Brennecka, M. C. Preul, W. D. Bichard and B. L. Vernon, World Neurosurg., 2012, 78, 469-480.

45 J. Mason, C. Dodge and G. Benndorf, Interv. Neuroradiol., 2018, 24, 574-579.

46 R. Pop, L. Mertz, A. Ilyes, D. Mihoc, J. S. Richter, M. Manisor, S. Kremer and R. Beaujeux, J. Neurointerv. Surg., 2019, 11, 706-709.

47 S. Lamin, H. S. Chew, S. Chavda, A. Thomas, M. Piano, L. Quilici, G. Pero, M. Holtmannspolter, M. E. Cronqvist, A. Casasco, L. Guimaraens, L. Paul, A. Gil Garcia, A. Aleu and R. Chapot, Am. J. Neuroradiol., 2017, 38, 127-131.

48 A. Helmy and N. Shaida, Cardiovasc. Intervent. Radiol., 2017, 40, 1094-1098.

49 S. S. Sirakov, A. Sirakov, K. Minkin, H. Hristov, K. Ninov, M. Penkov, V. Karakostov, K. Orlov, A. Gorbatykh, D. Kislitsin and R. Raychev, Interv. Neuroradiol., 2019, 25, 58-65. 
50 N. Kocer, H. Hanimoglu, S. Batur, S. G. Kandemirli, O. Kizilkilic, Z. Sanus, B. Oz, C. Islak and M. Y. Kaynar, Diagnostic Interv. Radiol., 2016, 22, 184-189.

51 D. F. Vollherbst, C. M. Sommer, C. Ulfert, J. Pfaff, M. Bendszus and M. A. Möhlenbruch, Am. J. Neuroradiol., 2017, 38, 1377-1382.

52 D. F. Vollherbst, C. M. Sommer, C. Ulfert, J. Pfaff, M. Bendszus and M. A. Möhlenbruch, Am. J. Neuroradiol., 2017, 38, 1377-1382.

53 O. Dudeck, O. Jordan, K. T. Hoffmann, A. F. Okuducu, I. Husmann, T. Kreuzer-Nagy, K. Tesmer, P. Podrabsky, H. Bruhn, J. Hilborn, D. A. Rüfenacht, E. Doelker and R. Felix, Am. J. Neuroradiol., 2006, 27, 1849-1855.

54 Antia Therapeutics S.A., EP2545085 (A1), 2011.

55 Z. Kulcsár, A. Karol, P. W. Kronen, P. Svende, K. Klein, O. Jordan and I. Wanke, Eur. Radiol., 2017, 27, 1248-1256.

56 G. Agusti, O. Jordan, G. Andersen, É. Doelker and Y. Chevalier, J. Appl. Polym. Sci., 2015, 132, 41791.

57 Antia Therapeutics S.A., US9434800B2, 2015.

58 G. Tamura, N. Kato, T. Yamazaki, Y. Akutsu, H. Hosoo, H. Kasuya and M. Sonobe, Neurol. Med. Chir., 2015, 55, 253-260.

59 H. Arakawa, Y. Murayama, C. R. Davis, D. L. Howard, W. L. Baumgardner, M. P. Marks and H. M. Do, Am. J. Neuroradiol., 2007, 28, 1191-1196.

60 R. J. Stewart, C. S. Wang, I. T. Song and J. P. Jones, Adv. Colloid Interface Sci., 2017, 239, 88-96.

61 J. P. Jones, M. Sima, R. G. O'Hara and R. J. Stewart, Adv. Healthcare Mater., 2016, 5, 795-801.

62 M. Johnson, Global Embolization Symposium and Technologies, 2018.

63 K. Y. Lee and D. J. Mooney, Prog. Polym. Sci., 2012, 37, 106-126.

64 T. A. Becker, M. C. Preul, W. D. Bichard, D. R. Kipke and C. G. Mcdougall, Neurosurgery, 2007, 60, 1119-1128.
65 B. P. Barnett, A. H. Hughes, S. Lin, A. Arepally and P. H. Gailloud, J. Vasc. Interv. Radiol., 2009, 20, 507-512.

66 L. Huang, M. Shen, R. Li, X. Zhang, Y. Sun, P. Gao, H. Fu, H. Liu, Y. He, Y. Du, J. Cao and Y. Duan, Oncotarget, 2016, 7, 73280-73291.

67 M. Bruchet and A. Melman, Carbohydr. Polym., 2015, 131, 57-64.

68 J. Cappello, J. Crissman, M. Crissman, F. Ferrari, G. Textor, O. Wallis, J. Whitledge, X. Zhou, D. Burman, L. Aukerman and E. Stedronsky, J. Controlled Release, 1998, 53, 105-117.

69 Z. Megeed, M. Haider, D. Li, B. W. O'Malley, J. Cappello and H. Ghandehari, J. Controlled Release, 2004, 94, 433-445.

70 A. Poursaid, R. Price, A. Tiede, E. Olson, E. Huo, L. McGill, H. Ghandehari and J. Cappello, Biomaterials, 2015, 57, 142-152.

71 H. Zhao, C. Zheng, G. Feng, Y. Zhao, H. Liang, H. Wu, G. Zhou, B. Liang, Y. Wang and X. Xia, AJNR. Am. J. Neuroradiol., 2013, 34, 169-176.

72 Y. Wang, N. Xu, Q. Luo, Y. Li, L. Sun, H. Wang, K. Xu, B. Wang and Y. Zhen, Interv. Neuroradiol., 2011, 17, 87-92.

73 X. Ning, C. Zhao, J. Pang, Z. Ding, Y. Wang, K. Xu, H. Chen, B. Li and Q. Luo, Exp. Ther. Med., 2015, 10, 316-322.

74 A. Salis, G. Rassu, M. Budai-Szucs, I. Benzoni, E. Csányi, S. Berkó, M. Maestri, P. Dionigi, E. P. Porcu, E. Gavini and P. Giunchedi, Expert Opin. Drug Delivery, 2015, 12, 1583-1596.

75 Y. Baba, M. Higashi and K. Awai, Minim. Invasive Ther. Allied Technol., 2018, 27, 17-21.

76 A. R. Cormier, X. Pang, M. I. Zimmerman, H.-X. Zhou and A. K. Paravastu, ACS Nano, 2013, 7, 7562-7572.

77 R. K. Avery, H. Albadawi, M. Akbari, Y. S. Zhang, M. J. Duggan, D. V. Sahani, B. D. Olsen, A. Khademhosseini and R. Oklu, Sci. Transl. Med., 2016, 8, 156-168. 